\section{Mosquito net as an environmental aid in the management of pemphigus}

\section{Ghazal Ahmed}

Department of Dermatology, Venereology, and Leprosy, All India Institute of Medical Sciences, Raipur, India

\section{Dear Editor}

Pemphigus is often difficult to manage disease, which involves, apart from the pharmacological management, maintaining the environmental temperature and humidity within a narrow range as the loss of skin easily result in thermal and hydration imbalance. ${ }^{1}$ While this might be easy to do this in a well-equipped hospital with airconditioning, but turns out to be difficult in a low resource set-up.

In this context, mosquito nets may play an adjunctive role. Using a mosquito net with appropriate coverage on the sides, keeping the upper surface open can help create a personal niche for the patient. It keeps the micro-environment around the patient warm and comfortable and can even possibly prevent fluid loss from extensive erosions as it is well-known for attenuating the airflow. ${ }^{2}$ Very often, patients with exten- sive erosions need open dressing with minimal clothing for faster healing. Nevertheless, the privacy of the patient should be regarded as right and respected. ${ }^{3}$ The mosquito net-based set-up provides a private space for the patient to avoid unnecessary anxiety of nakedness. While there might be some alternative to the mosquito net, we have used the net considering the wide availability, cheap, and even customizable. There is limited or no data available on the ideal micro-environment for pemphigus treatment. The pemphigus resembles burn patients in terms of skin loss and evaporative loss. The recommended temperature of 32-35 degrees Celsius with a relative humidity of $50 \%$ for burn patient management to reduce the evaporative loss ${ }^{4}$ might be applied to the pemphigus management. Airflow around the burn patient is also advocated in burn patients in the exposure method. However, mosquito net mesh size is found to be inversely related to airflow. To overcome this problem, we have covered the sides of the net with cloths and kept the top uncovered. The mosquito net can also be stitched in a customized way using smaller mesh sides but having a more oversized mesh top. A ceiling fan can be placed just above the net. Temperature is also another critical factor, ${ }^{5}$ and in the winter or colder places will need attention. We have used a 100-200-Watt tungsten fila-

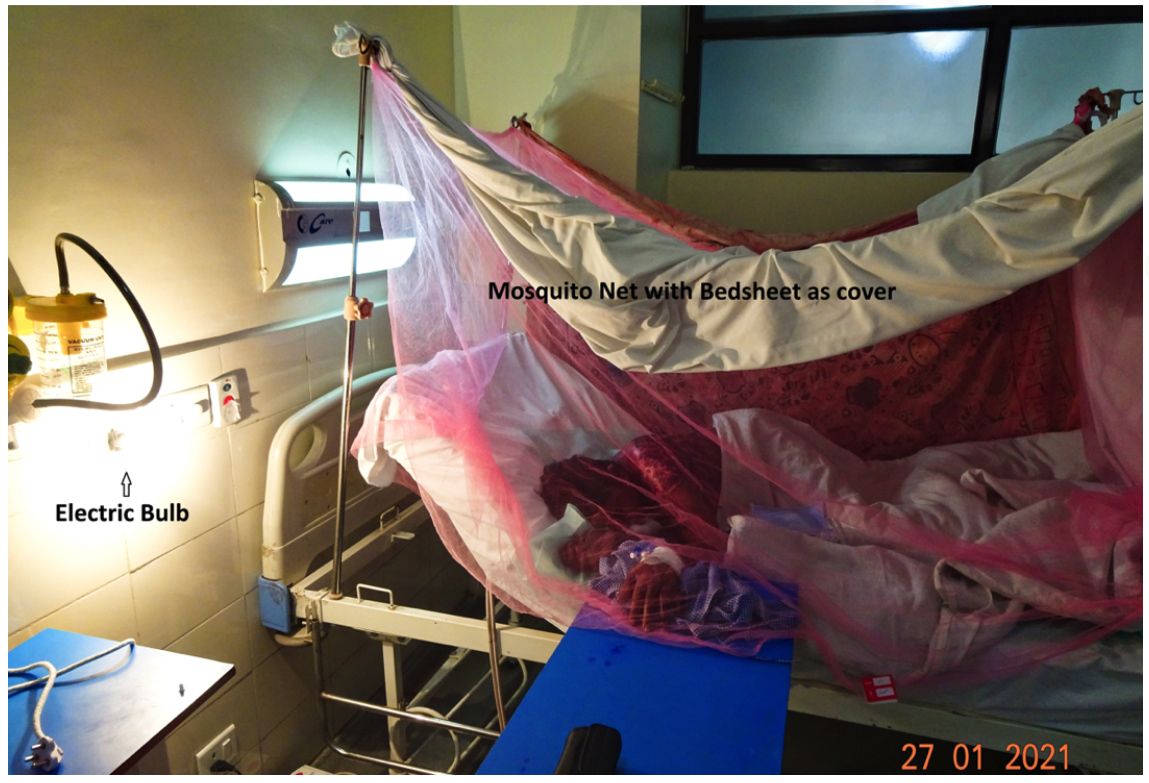

Figure 1. Showing a patient inside a mosquito net with bedsheets hanging from sides and an incandescent bulb. In this picture, bedsheets were folded up to show the set-up.
Correspondence: Ghazal Ahmed, Department of Dermatology, Venereology, and Leprosy, Block-D, First Floor, All India Institute of Medical Sciences, PIN 492099, Raipur, India. Tel.: +91.9835521686

E-mail: ghazal.ahmed4u@gmail.com

Key words: Bullous disease; pemphigus; body temperature regulation; moisture.

Conflict of interests: The author declares no conflict of interest.

Availability of data and materials: All data underlying the findings are fully available.

Received for publication: 24 April 2021.

Revision received: 24 August 2021.

Accepted for publication: 13 December 2021.

This work is licensed under a Creative Commons Attribution 4.0 License (by-nc 4.0).

C Copyright: the Author(s), 2022

Licensee PAGEPress, Italy

Healthcare in Low-resource Settings 2022; 10:9828 doi:10.4081/hls.2022.9828

ment bulb on the open side if needed to add heat. However, other external heating sources can also be used. Nevertheless, we will require clinical studies to evaluate the system objectively and in the clinical outcome context.

\section{References}

1. Kar PK. Recent trends in the management of pemphigus vulgaris. Med J Armed Forces India 1998;54:243-6.

2. von Seidlein L, Ikonomidis K, Bruun R, et al. Airflow attenuation and bed net utilization: observations from Africa and Asia. Malar J 2012;11:200.

3. Lindsay E, Renyi R, Wilkie P, et al. Patient-centred care: a call to action for wound management. J Wound Care 2017;26:662-77.

4. Martin CJ, Ferguson JC, Rayner C. Environmental conditions for treatment of burned patients by the exposure method. Burns 1992;18:273-82.

5. Alonso Fernández JM, López Pablo C. Body temperature and heating temperature in the care of large burn patients. Global Nursing 2021;20:466-88. 